\section{Personalizing cystic fibrosis in vitro}

\section{By Chris Cain, Senior Writer}

A team at University Medical Center Utrecht has developed a rapid and quantitative in vitro intestinal cell-based assay for CFTR function that could help prospectively identify responders to targeted cystic fibrosis therapies. ${ }^{1}$ The researchers are now planning to test how well the approach can predict drug responses in the clinic.

Cystic fibrosis is caused by inherited mutations that reduce the function of the cystic fibrosis transmembrane conductance regulator (CFTR), an anion channel that helps keep the lung and intestinal epithelium hydrated and prevents the mucus buildup that leads to airway obstruction and infection.

The only marketed disease-modifying treatment for CF is Kalydeco ivacaftor, a small molecule CFTR potentiator from Vertex Pharmaceuticals Inc. that increases chloride transport through the channel. The drug was approved last year and is indicated to treat only the $4 \%$ of patients with CF carrying the G551D CFTR mutation.

Additional compounds from Vertex are in late-stage development to treat patients carrying $\triangle \mathrm{F} 508 \mathrm{CFTR}$, the dominant disease-associated mutation, carried by about two-thirds of patients. These include VX809 and VX-661, which are related compounds that act directly on mutant CFTR to correct its structure.

This year, VX-809 in combination with Kalydeco entered Phase III testing to treat patients carrying the $\Delta$ F508 CFTR mutation. VX-661 is in Phase II testing with Kalydeco in the same population.

Despite these recent clinical advances, a significant fraction of patients with $\mathrm{CF}$ are not eligible for existing treatments because they carry rare mutations in which the efficacy of CFTR-targeted compounds has not been confirmed.

Even for those patient populations addressed by existing treatments, multiple academic teams and companies are seeking to develop additional compounds and combination therapies that will further improve CFTR function. ${ }^{2}$

The development and testing of CFTRtargeted compounds has been hampered by a lack of robust in vitro assays to quantify their activity. The gold standard for preclinical testing relies on cultured bronchial lung epithelial cells taken from lung explants. ${ }^{3}$ However, access to these bronchial cells is limited because it requires sampling of patient tissue obtained after a lung transplant or biopsy, and their replicative potential is limited.
Instead of primary cells, many phenotypic screens rely on cell lines engineered to express CFTR mutants. But these cells lines are less physiologically relevant than patients' bronchial cells. Indeed, Jeffrey Beekman, principal investigator of pediatric pulmonology at UMC Utrecht, told SciBX the lack of more accessible primary cell models has hindered the development and testing of CF treatments.

"A big issue for drug screening is to have a relevant cell model, and the field has generally agreed that it is important to move to primary cells as quickly as possible," he said. "False positives are commonly identified in cell line-based screening approaches. Up to $90 \%$ of hits in these cells could not be validated in primary cells, and there may be false negatives that are simply missed."

Thus, Beekman teamed up with Hans Clevers, professor of molecular genetics at the Hubrecht Institute and president of the Royal Netherlands Academy of Arts and Sciences, to develop a more accessible primary cell culture model for CFTR function.

The starting point for the model was a recent culture technique developed in Clevers's lab that enabled intestinal stem cells to be grown into organoids that recapitulate the phenotype of in vivo intestinal tissue architecture. ${ }^{4,5}$ "These primary adult stem cell cultures can be cultured for long times in vitro without genetic modifications and can be biobanked," said Beekman. To measure CFTR function in these organoids, the team treated them with forskolin, a commonly used pharmacological tool that activates the transporter by raising intracellular cyclic AMP (cAMP) levels.

By activating CFTR, forskolin induces fluid transport across the organoid's intestinal epithelial barrier. The results were immediate and striking. "In organoids derived from mice, after 40 minutes some just pop because they have so much fluid and tension in them due to the amount of fluid transport. We were amazed by the quantity and the speed of the response and the complete dependency on CFTR," Beekman said.

Because the effect is dependent on CFTR function, organoids expressing defective CFTR should have less forskolin-induced swelling than those expressing normal CFTR. Compounds or conditions that improve CFTR function should restore the normal swelling response.

Indeed, in organoids derived from mouse intestinal cells, treatment with CFTR inhibitors or use of cells lacking Cftr or carrying $\Delta$ F508 $\mathrm{Cftr}$ reduced the forskolin-induced swelling compared with vehicle or

\section{"This quantification method is straightforward and fully compatible with high throughput screens." - Jeffrey Beekman, University Medical Center Utrecht} wild-type controls. In $\Delta$ F508 Cftr organoids, corrector compounds increased forskolininduced swelling compared with vehicle.

Beekman said the results were highly quantitative and provided a large signal-to-noise ratio that allowed the relative effects of different compounds to be measured.

"We plate 30-70 organoids per well, in triplicate, at several time points. More than $90 \%$ of the organoids in a single well respond quickly by swelling, allowing a uniform and accurate measurement between the three wells. The measurements are quantified by fluorescently labeling the organoids, and their relative increase is measured by confocal microscopy and quantified by image 
analysis of a series of images within a time frame of typically 60 minutes for human structures," he said. "This quantification method is straightforward and fully compatible with high throughput screens."

Having optimized the approach, the team next applied it to patient samples to determine its clinical relevance. In organoids derived from rectal biopsy tissue from patients with $\mathrm{CF}$, those carrying CFTR mutations associated with mild defects in CFTR function had mild reductions in forskolin-induced swelling compared with healthy controls. Organoids from patients with mutations associated with severe defects in CFTR function, such as those homozygous for $\triangle$ F508 CFTR, had highly reduced swelling.

Finally, the team examined the effect of CFTR potentiators and correctors in the model. In samples homozygous for $\triangle \mathrm{F} 508 \mathrm{CFTR}$, combinations of compounds, including Kalydeco plus VX-809, improved CFTR function compared with individual compounds or vehicle controls. Organoids from individual patients showed highly reproducible responses, but responses varied among patient samples, suggesting the assay may be able to predict variation in response to the drug.

Results were published in Nature Medicine.

\section{Clinical correlations}

CF researchers and industry scientists agreed the assay could be a powerful tool for compound validation and said it has the potential to guide clinical-trial design if the results are found to correlate with patient response.

Philip Thomas, professor of physiology at The University of Texas Southwestern Medical Center, told SciBX the new assay will have a key role in validating compounds in different CFTR mutant backgrounds. "It was a beautifully executed comprehensive study where they looked at inhibitors, correctors and activators," he said. "In principle it could be used as a primary screen, but the immediate application will be as a secondary assay for compound hits that are identified by screening in cell lines."

He added that the assay will be of particular use for predicting the response of patients with CF carrying rare mutations in CFTR. "I would guess that this will open up a whole host of rare genotypes for testing to see if they respond to Kalydeco," said Thomas. "For some of these patients, you have to realize that there might not be primary bronchial epithelial cells available because if you have less severe disease it's unlikely you are going to get a lung transplant."

In his own lab, Thomas, who is also a founder of Reata Pharmaceuticals Inc., is studying CFTR folding and the mechanism of action of corrector compounds.

Fred Van Goor, head of biology for Vertex's CF research program, said the key advantage of the approach is its ease of application. "The one advantage that I see is that it is easier to look at specific genotypes of interest. We use cultured airway cells derived from patients with CF, but this approach could extend those studies. We have fairly effectively studied the effect of specific mutations in recombinant cell lines, but this is a nice way to study situations where there are multiple mutations."

Vertex has published preclinical work demonstrating the effect of Kalydeco in human bronchial epithelial cells in patients with other CFTR gating mutations besides G551D. ${ }^{6}$ Last year, the company began a Phase III trial of Kalydeco in patients carrying the R117H mutation in CFTR.
David Weiner, CMO of Proteostasis Therapeutics Inc., agreed the new model could be relevant for patients carrying rare CF mutations. "One of the tremendous potential applications here is if our compounds or other correctors can be developed beyond the $\Delta \mathrm{F} 508$ mutant population. It can be difficult to do randomized controlled trials with such small numbers of patients, so biological surrogate measures for clinical efficacy are particularly important."

Proteostasis President and CSO Peter Reinhart agreed and said the next steps will include validation of the model in additional laboratories and a comparison of results in the in vitro assay with in vivo patient response. "I think this assay is promising because it provides another way of establishing a set of correlations between preclinical data sets and clinical efficacy," he said.

The company has small molecule proteostasis modulators in lead optimization to treat patients who have $\triangle$ F508 CFTR.

Beekman now is using the organoid assay for $\mathrm{CF}$ diagnosis and to assess CFTR genotypes that are responsive to CFTR-restoring drugs such as Kalydeco and VX-809 in vitro. "We have the biggest clinical CF center in the Netherlands with about 400 patients with CF, and collaborate tightly with the CF centers in Rotterdam and The Hague. We are trying to see if we can make predictions about CF disease progression and development using this assay, and we are also trying to set up studies to show that drug efficacy in vitro also relates to drug efficacy in vivo," he said.

He said his lab has easy access to patient samples because it is standard procedure in the centers of Utrecht, Rotterdam and The Hague for patients newly diagnosed with CF to undergo a rectal section biopsy at 12 months old to measure the function of CFTR using electrical current ex vivo. The rectal biopsies can then be transported and organoid cultures started without affecting the diagnostic procedure.

Beekman also is collaborating with Gergely Lukacs, professor of physiology at McGill University, to test new combinations of corrector compounds in the assay. ${ }^{7}$

Clevers and Beekman have filed for patents covering the work, and the IP is available for licensing. Beekman said he is involved with setting up a not-for-profit foundation to handle the licensing of this and other assays using cultured organoids.

Cain, C. SciBX 6(23); doi:10.1038/scibx.2013.564

Published online June 13, 2013

REFERENCES

1. Dekkers, J.F. et al. Nat. Med.; published online June 2, 2013; doi:10.1038/nm.3201

Contact: Jeffrey M. Beekman, University Medical Center Utrecht, the Netherlands

e-mail: jbeekman@umcutrecht.nl

2. Lukacs, G.L. \& Verkman A.S. Trends Mol. Med. 18, 81-91 (2012)

3. Neuberger, T. et al. Methods Mol. Biol. 741, 39-54 (2011)

4. Sato, T. et al. Gastroenterology 141, 1762-1772 (2011)

5. Sato, T. \& Clevers, H. Science 340, 1190-1194 (2013)

6. Yu, H. et al. J. Cyst. Fibros. 11, 237-245 (2012)

7. Okiyoneda, T. et al. Nat. Chem. Biol.; published online May 12, 2013; doi:10.1038/nchembio.1253

Contact: Gergely L. Lukacs, McGill University, Montreal, Quebec, Canada

e-mail: gergely.lukacs@mcgill.ca

COMPANIES AND INSTITUTIONS MENTIONED

Hubrecht Institute, Utrecht, the Netherlands 


\section{ANALYSIS}

\section{COVER STORY}

McGill University, Montreal, Quebec, Canada

Proteostasis Therapeutics Inc., Cambridge, Mass.

Reata Pharmaceuticals Inc., Irving, Texas

Royal Netherlands Academy of Arts and Sciences, Amsterdam, the Netherlands
University Medical Center Utrecht, Utrecht, the Netherlands The University of Texas Southwestern Medical Center, Dallas, Texas

Vertex Pharmaceuticals Inc. (NASDAQ:VRTX), Cambridge, Mass. 